\title{
Tendências do Ensino de Anatomia Animal na Graduação de Medicina Veterinária
}

\author{
Catia Helena de Almeida Lima Massari ${ }^{1}$, Luciana Silveira Flores Schoenau ${ }^{1}$, Andressa \\ Daronco Cereta ${ }^{1}$, Maria Angélica Miglino ${ }^{1,2 *}$
}

${ }^{1}$ Programa de Pós-Graduação em Anatomia dos Animais Domésticos e Silvestres da Faculdade de Medicina Veterinária e Zootecnia da Universidade de São Paulo

${ }^{2}$ Departamento de Cirurgia da Faculdade de Medicina Veterinária e Zootecnia da Universidade de São Paulo

* Autora para correspondência: miglino@usp.br

\begin{abstract}
RESUMO
O ensino de anatomia é de fundamental importância a todos os profissionais da área da saúde. Especialmente no campo da macroscopia, observam-se estímulos para o desenvolvimento de novos conceitos de educação médica com a aplicação de diferentes abordagens pedagógicas. Nos cursos de graduação em Medicina Veterinária, o método tradicional de ensino vem passando por inovações semestre após semestre. O presente artigo aborda quatro tendências no ensino de anatomia veterinária, verificadas através de uma revisão crítica: a) Aprendizagem assistida por computador a fim de desenvolver modelos anatômicos de animais virtuais; b) Modelos anatômicos artesanais, manequins e simuladores; c) Gravação de videoaulas e compartilhamento pela internet; e d) Uso de técnicas de preservação de cadáveres livres de formol. Conclui-se que a melhor metodologia de ensino de anatomia veterinária tende a ser aquela em que exista a associação de todos os novos recursos com a dissecação animal, complementando-se uns aos outros.
\end{abstract}

Palavras-Chave: Ensino Superior; Medicina Veterinária; Anatomia Animal; Objetos Educacionais de Aprendizagem; Peças Anatômicas.

\begin{abstract}
Anatomy teaching has fundamental importance to all health professionals. Especially in the field of gross anatomy, incentives have been observed for the development of new concepts of medical education with the application of different pedagogical approaches. In Veterinary Medicine courses, the traditional teaching method has undergone countinuous innovations semester after semester. The present paper aims to investigate four trends in veterinary anatomy teaching through a critical review about: a) Computer-assisted learning in order to develop anatomical models of virtual animals; b) Anatomical models, mannequin and simulators; c) Videotapes recording and internet sharing; and d) Use of formaldehyde-free cadaver preservation techniques. Therefore, it concluded that the best methodology of veterinary anatomy teaching tends to be the association of all these new resources with animal dissection, complementing each other.
\end{abstract}

Keywords: Higher Education; Veterinary Medicine; Animal Anatomy; Educational Learning Objects; Anatomical Pieces.

\section{Introdução}

O ensino de anatomia revela-se importante para todos os profissionais da área da saúde - seja humana ou animal. De modo mais específico, observam-se, sobretudo no campo da macroscopia, estímulos para o desenvolvimento de novos conceitos de educação médica com a pesquisa e aplicação de novas abordagens pedagógicas. $\mathrm{Ou}$ seja, sendo o objetivo da anatomia macroscópica o estudo das estruturas corpóreas observáveis a olho nu, atualmente muitos recursos tecnológicos dos mais variados possíveis estão sendo incorporados à práxis laboratorial (MCGUSKEY et al., 2005).

$\mathrm{Na}$ maioria das faculdades de Medicina Veterinária, as disciplinas relacionadas à anatomia (descritiva, sistêmica, topográfica e cirúrgica) vêm tendo suas cargas horárias reduzidas nos últimos 
anos. Com isso, há um compromisso dos docentes em identificar e selecionar material didático em quantidade e qualidade satisfatória ao ensino superior (DYCE et al., 2010).

Nos cursos de graduação (bacharelado) em Medicina Veterinária, uma nova geração de estudantes já está se formando tendo experimentado práticas complementares ao método tradicional, possibilitando cada vez mais criatividade acadêmica e transformação curricular. Mas, quais seriam essas ferramentas didáticas alternativas que poderiam ser utilizadas no ensino de anatomia animal? Inúmeros modelos, manequins e simuladores estão sendo desenvolvidos artesanalmente nas salas de aula ou até mesmo comercialmente por algumas empresas nacionais. Os programas de aprendizagem assistida por computador possibilitam simulações multimídias através de dissecações virtuais; softwares interativos podem gerar imagens 3D que propiciam uma imersão do estudante em habilidades e procedimentos psicomotores a serem praticados de forma altamente sensorial, até mesmo tendo oportunidades de interação em tempo real ou impressão tridimensional de peças anatômicas. Os vídeos sobre anatomia são encontrados de forma rápida pela internet, confrontando-se cada vez mais com a disponibilidade do aluno em aprender através de prossecções de peças cadavéricas no laboratório. Nas aulas práticas, o uso do formol torna-se desconfortável devido aos riscos para a saúde dos alunos, funcionários e professores dentro do laboratório, o que desafia o docente a buscar novas técnicas para a conservação de peças anatômicas. Contudo, o estudo da anatomia em Medicina Veterinária não seria completo sem o trabalho prático com cadáveres de animais e seus tecidos; para isso, buscam-se sempre materiais de origem ética, melhores soluções para a preservação dos espécimes e maior cooperação entre departamentos que possam tornar-se fontes de materiais didáticos dentro do meio acadêmico, como o de patologia e cirurgia (MARTINSEN \& JUKES, 2005; THEORET et al., 2007).

\section{Breve Descrição do Método Tradicional de Ensino de Anatomia Veterinária}

O ensino de Anatomia objetiva abordar a forma, a disposição e a estrutura dos tecidos e órgãos que compõem o corpo. Em sua etimologia, a palavra, de origem grega, significa literalmente "cortar em pedaços", uma vez que a dissecação de cadáveres é o método tradicionalmente utilizado no processo de ensino-aprendizagem desse ramo do conhecimento médico veterinário (DYGE et al., 2010).

Historicamente, a dissecação de cadáveres é considerada a referência de qualidade para os ensinamentos de anatomia macroscópica. $\mathrm{O}$ debate acerca da educação em anatomia é vasto, e ponderações a respeito de vantagens e desvantagens do método tradicional de ensino são suscitadas nos dias de hoje (WILSON et al., 2017).

Apesar dos muitos desafios relacionados ao uso de material cadavérico para ensinar estudantes de Medicina Veterinária, a abordagem tradicional do ensino, com bisturi em mãos no laboratório, continua sendo um passo importante à instrução dos alunos (GUMMERY et al., 2017).

\section{Aprendizagem Assistida por Computador: Modelos Anatômicos de Animais Virtuais}

$\mathrm{O}$ ensino da anatomia veterinária vem sendo repensado nos últimos anos, buscando-se introduzir novas tecnologias que substituam o tradicional método baseado em cadáveres fixados, mantendo a qualidade do aprendizado. Tal complementação visa a atender anseios das comissões de ética no uso de animais (GEUA), além de perpassar por possíveis questões ambientais reduzindo a geração de resíduos químicos e preservando a saúde dos indivíduos envolvidos nessas práticas (SIMÃO \& DE MELO, 2015).

A tecnologia do diagnóstico por imagem está se revelando um importante recurso de ensino em anatomia animal na medida em que se torna mais avançada e amplamente disponível para pacientes veterinários com a crescente popularização da tomografia computadorizada e da ressonância magnética incrementando a capacidade espacial 
e visual dos estudantes de Medicina Veterinária (DELISSER \& CARWARDINE, 2017).

Verifica-se que, na disciplina de anatomia animal, os tópicos são muitas vezes abordados a partir de diagramas bidimensionais (2D), fotografias e desenhos de atlas veterinários que mostram apenas um ponto de vista fixo. Isso leva à perda de informações espaciais, podendo induzir no estudante confusão ao transferir esse conhecimento ao paciente vivo. Assim, os métodos de aprendizagem assistidos por computador como modelos anatômicos 3D interativos têm ganhado espaço na educação em Medicina Veterinária (RAFFAN et al., 2017; SCHOENFELD-TACHER et al., 2017).

Os modelos anatômicos tridimensionais (3D), através de aprendizagem assistida por computador, recebem cada vez mais refinamento devido aos recursos derivados do escaneamento da superfície de objetos reais (como o esqueleto animal) ou do mapeamento de regiões anatômicas de interesse a partir de imagens de secções em série do corpo real. Nesses modelos, o aluno pode controlar a posição da imagem como se fosse um objeto 3D real em suas mãos, tendo noção de medidas e profundidades para aprendizagem de anatomia espacial, além de poder imprimir tais imagens através de técnicas de prototipagem em impressoras específicas (GARG et al., 2001; GARG et al., 2002).

Ademais, na área de anatomia microscópica, o advento de tecnologias digitais adotadas em sala de aula como ferramentas para visualização e interação com o conteúdo a ser ministrado já é realidade nos cursos de graduação com a utilização de laminários virtuais (SIVIERO \& DE OLIVEIRA, 2016; RINALDI et al., 2017). Também se faz disponível o uso de simuladores de células específicas para elucidação de certos eventos fisiológicos como, a título de exemplo, em circuitos neurais (PETTO et al., 2017).

Ainda, possibilita dissecar virtualmente algumas regiões extremamente frágeis ou de difícil acesso nas peças anatômicas reais e melhora o ensino a respeito de interações espaciais complexas.
Também a impressão 3D pode ser empregada para reproduzir diversos objetos, incluindo próteses e esboços de peças anatômicas de diversas espécies animais (HARRISON et al., 2001; BIASUTTO et al., 2006; CHITTARO \& RANON, 2007; JOHN, 2007; HISLEY et al., 2008; SEIXAS-MIKELUS et al., 2010; CODD \& CHOUDHURY, 2011; BATTULGA et al., 2012; PREEGE et al., 2013; MCMENAMIN et al., 2014; SALTARELLI et al., 2014; VIEHDORFER et al., 2014; SIMÃO \& DE MELO, 2015; YAMMINE \& VIOLATO, 2015; NEMANIC et al., 2016; SABER et al., 2016; DOS REIS et al., 2017; GROSS et al., 2017; RINALDI et al., 2017; SCHOENFELD-TACHER et al., 2017; SMITH, et al., 2018).

\section{Modelos Anatômicos, Manequins e Simu- ladores Manufaturados}

No Brasil, atualmente já existem empresas que comercializam simuladores veterinários em tamanho natural para estudo e treinamento de diversas técnicas em animais (GIVIAM, 2018). Da mesma maneira, ossos artificiais fabricados em poliuretano rígido já são comercializados para utilização em aulas práticas a respeito de anatomia descritiva de algumas espécies de animais domésticos como caninos, felinos e equinos (NACIONAL OSSOS, 2018).

No entanto, destaque aqui é dado à confecção artesanal de modelos anatômicos, como, por exemplo, a representação das articulações do ombro e do joelho de cão, utilizando massa de biscuit para reproduzir os diferentes ligamentos existentes nas referidas articulações, ou a representação da topografia vascular da face de um equino demonstrada com a colagem de lã colorida sobre a superfície óssea da cabeça do animal, ilustrando dessa forma toda a trama arterial que é responsável pela irrigação sanguínea dessa região (DE MORAES SOUZA et al., 2012).

A modelagem com argila também é uma dessas alternativas que fornecem uma solução cinestésica e tridimensional numa abordagem construtiva e sensorial para aprender anatomia. Acredita-se que, além de despertar a atenção do 
estudante para tal tarefa, certamente aumente a carga cognitiva no momento da realização dessa atividade (DEHOFF et al., 2011).

\section{Videoaulas Compartilhadas pela Internet}

A gravação de videoaulas sobre anatomia macroscópica e seu compartilhamento através da internet tentam "driblar" a redução de horas-aula em laboratório que vem ocorrendo na maioria das faculdades: disponibiliza-se aos estudantes material visual com boa qualidade para estudos em casa (TOPPING, 2014).

Embora seja controverso que as videoaulas efetivamente melhorem o desempenho acadêmico nas disciplinas relacionadas à anatomia animal, uma vez que jamais substituem o estudo presencial no laboratório, muitos estudantes são favoráveis à sua aplicação (THEORET et al., 2007; SAXENA et al., 2008; MAHMUD et al., 2011). Schoenau e Schoenau (2013), fazendo uso da produção de videoaulas pelos acadêmicos como forma de aprendizado, nas quais estes participaram ativamente do processo, concluíram que o uso dos vídeos na disciplina de anatomia animal constitui uma ferramenta de suporte divertida, que promove satisfação pessoal, integração do grupo, divisão de tarefas e responsabilidade. Além do mais, estimula o aprendizado através da experiência. No entanto, alerta-se que a disponibilidade de tempo, do suporte tecnológico e do material didático anatômico deve ser levada em consideração.

Certamente, essa demanda é sentida por muitos docentes em anatomia veterinária nos dias de hoje, quando os alunos tendem a estudar para as avaliações também através de redes sociais on-line e sites de compartilhamento de vídeos como o You Tube, o qual demonstra ser um recurso emergente para a educação em anatomia (JAFFAR, 2012).

\section{Técnicas de Preservação de Cadáveres Animais sem o Uso de Solução de Formol}

Diversas são as técnicas anatômicas que se destinam à preservação de cadáveres para o ensino (RODRIGUES, 2005). Desde que foi descoberto no século XVII, o formaldeído (aldeído fórmico, formol ou formalina) é, sem dúvida, o fixador e conservante de tecido biológico mais utilizado em laboratórios de anatomia, por apresentar ação bactericida, fungicida, virucida e esporicida. No entanto, oferece riscos de carcinogenicidade, especialmente como doença ocupacional a docentes, discentes e funcionários submetidos à exposição tóxica no laboratório. Por também ser tóxico ao meio ambiente, não pode ser descartado no esgoto comum, devendo ser retirado através de serviço de firma especializada na coleta adequada de resíduos químicos (LIMBERGER et al., 2011; NETO \& BIGONI, 2014).

Franceschini e Carvalho (1993) relatam que a utilização dessa solução é indevida na maioria dos laboratórios de anatomia, tendo em vista que muitos a usam para a conservação dos cadáveres e não apenas para a fixação dos mesmos. Isso ocorre, geralmente, pelo fato de que a solução de formol a $10 \%$ é fácil de ser preparada e também apresenta um menor custo aos laboratórios.

Conhecer tais riscos é primordial para que o popular formol possa ser substituído por outras técnicas de conservação como a glicerinação e a plastinação, que oferecem menor vulnerabilidade à saúde. No entanto, verifica-se que, embora estas sejam técnicas muito eficientes para a conservação de peças anatômicas, principalmente para fins de estudo em aulas práticas por evitarem riscos à saúde, seus custos ainda são elevados, e isso explica a pouca utilização da glicerinação e da plastinação em laboratórios de anatomia animal (CURY et al., 2013; NETO \& BIGONI, 2014).

Historicamente, em 1998, numa viagem a estudo à Universidade de Guarulhos pelo Curso de Pós-Graduação em Anatomia dos Animais Domésticos e Silvestres da Faculdade de Medicina Veterinária da Universidade de São Paulo, o professor Dr. Alzido de Oliveira (in memoriam) apresentou uma técnica de conservação de cadáveres desenvolvida e utilizada naquela instituição por ele: a solução de Laskowski modificada. Tal solução foi implantada no Laboratório de Anatomia Animal da Universidade Federal de Santa Maria - UFSM 
em 2002 e continua sendo utilizada até os dias de hoje com ótimos resultados ${ }^{1}$. Nessa técnica, as peças são acondicionadas em meio líquido, havendo na composição: a glicerina e o álcool como agentes conservadores, o ácido bórico com ação fungicida, e o ácido fênico com ação bactericida.

Outra técnica de preservação de cadáveres é através da solução Larssen. Criada no século XVIII, foi adaptada para reduzir os custos do produto sem perder a qualidade (solução de Larssen modificada). Tal líquido, quando perfundido, consegue preservar os tecidos, conservando a cor e a flexibilidade características das peças anatômicas. Nessa técnica, o cadáver é mantido no freezer, sendo descongelado a cada uso (DA SILVA et al., 2004; MATERA, 2009).

Oliveira $(2014 ; 2017)$, pensando na substituição do formaldeído devido a seus diversos efeitos nocivos, desenvolveu uma técnica alternativa de conservação de cadáveres em meio líquido, que consiste no uso de uma solução salina (cloreto de sódio a 30\%), utilizada também até os dias de hoje nos laboratórios de anatomia da Universidade Estadual Paulista - Unesp e da Universidade de São Paulo - USP.

Ainda, visando a abolir o uso do formol como conservante e permitindo assim um ambiente agradável para ensino-aprendizagem, outras técnicas anatômicas como a criodesidratação, a injeção de látex e a injeção de vinilite seguida de corrosão são executadas, utilizando corpos e órgãos de animais provenientes de doações. A técnica de criodesidratação consiste em desidratar as peças para serem utilizadas em sala de aula (TEIXEIRA et al., 1991; TEIXEIRA FILHO, 1996). As técnicas de preenchimento consistem em injetar em vasos sanguíneos, linfáticos, biliares e até em vias urinárias, reprodutoras e brônquios o látex natural, borracha siliconada e polímeros como o acetato de vinila - produtos de maior aceitação pelos anatomistas devido ao alto grau de penetração que fazem em pequenos capilares (CURY et al., 2013).

Já na técnica de plastinação, após a dissecação do cadáver, este é submerso em acetona pura, para que todo o líquido e as gorduras do corpo sejam removidos e, em seguida, a peça deve ser submetida ao processo de vácuo para substituir a acetona do corpo por um polímero (LATORRE et al., 2007; ARREDONDO et al., 2016; FRUHSTORFER et al., 2011).

\section{Considerações Finais}

Mesmo com a consciência de que as inovações no ensino de anatomia através do uso de metodologias computadorizadas ou manufaturadas desenvolvam um novo campo para pesquisa, oferecendo aos estudantes de Medicina Veterinária muitos elementos para facilitar a abordagem das estruturas anatômicas, acredita-se que a aula prática no laboratório em que o discente experimenta o contato direto com as peças de cadáveres e seus tecidos ainda não possa ser substituída.

Salienta-se que a solução de formol ainda se faz necessária para a fixação das peças numa primeira etapa da preparação anatômica, embora felizmente seu uso já não seja obrigatório para a conservação dessas numa segunda etapa. Nessa segunda etapa, diante das diferentes técnicas oferecidas, devem-se analisar custos, forma de descarte dos resíduos, praticidade de uso, odor emanado no ambiente, coloração final das peças, maleabilidade e aceitação pelos estudantes, para uma escolha adequada do meio de conservação das peças nos laboratórios.

Conclui-se, portanto, que a melhor metodologia de ensino de anatomia veterinária tende a ser aquela em que exista a associação de todos os novos recursos com a dissecação animal, complementando uns aos outros.

\section{Nota}

${ }^{1}$ Comunicação pessoal da Profa. Dra. Luciana Silveira Flores Schoenau, em 16 de fevereiro de 2018.

\section{Referências Bibliográficas}

AL-KHALILI, Sereen M.; COPPOG, Gordon L. $2 \mathrm{D}$ and $3 \mathrm{D}$ stereoscopic videos used as pre-anatomy lab tools improve students' examination performance 
in a veterinary gross anatomy course. Journal of veterinary medical education, v. 41, n. 1, 2014, pp. 68-76.

ARREDONDO, Jorge; LÓPEZ-ALBORS, Octavio; RECILLAS, Sergio; VICTORIA, Mauro; CASTELÁN, Octavio; GONZÁLEZ-RONQUILLO, Manuel; BECERRIL, Sigrid \& LATORRE, Rafael. Modelo Virtual Tridimensional de la Articulación Cubital del Perro a Partir de Cortes Plastinados Ultradelgados. International Journal of Morphology, v. 34, n. 4, 2016, pp. 1253-1258.

BATTULGA, Bayanmunkh; KONISHI, Takeshi; TAMURA, Yoko \& MORIGUCHI, Hiroki. The effectiveness of an interactive 3-dimensional computer graphics model for medical education. Interactive journal of medical research, v. 1, n. 2, 2012.

BIASUTTO, Susana Norma; CAUSSA, Lucas Ignacio; DEL RÍO, Luis Esteban Criado. Teaching anatomy: cadavers vs. computers?. Annals of Anatomy-Anatomischer Anzeiger, v. 188, n. 2, 2006, pp. 187-190.

CHITTARO, Luca; RANON, Roberto. Web3D technologies in learning, education and training: Motivations, issues, opportunities. Computers \& Education, v. 49, n. 1, 2007, pp. 3-18.

CIVIAM. Simuladores Veterinários. Disponível em: <http://www.civiam.com.br/civiam/index.php/ simulacao-veterinaria.html $>$. Acesso em 15 jan. 2018.

CODD, Anthony M.; CHOUDHURY, Bipasha. Virtual reality anatomy: Is it comparable with traditional methods in the teaching of human forearm musculoskeletal anatomy?. Anatomical sciences education, v. 4, n. 3, 2011, pp. 119-125.

GURY, Fabio Sergio; CENSONI,Julia Barrionuevo; AMBRÓSIO, Carlos Eduardo. Técnicas anatômicas no ensino da prática de anatomia animal. Pesquisa Veterinária Brasileira, v. 33, n. 5, 2013, pp. 688-696.

DA SILVA, Rosane Maria Guimarães; MATERA, Julia Maria; RIBEIRO, Antonio Augusto Coppi Maciel. Preservation of cadavers for surgical technique training. Veterinary surgery, v. 33, n. 6, 2004, pp. 606-608.

DE MORAES SOUZA, Aline Luize; DE ASSUMÇÃO, Rômulo Ferreira; GUIMARÃES, Luísa Faustino \& RODRIGUES, Ana Bárbara Freitas. Utilização de métodos didáticos alternativos para o estudo da anatomia veterinária. PUBVET, v. 6, 2012, pp. Art. 1423-1428.

DEHOFF, Mary Ellen; CLARK, Krista L.; MEGANATHAN, Karthikeyan. Learning outcomes and student-perceived value of clay modeling and cat dissection in undergraduate human anatomy and physiology. Advances in physiology education, v. 35, n. 1, 2011, pp. 68-75.

DELISSER, Peter J.; CARWARDINE, Darren. Student Perceptions of Sectional CT/MRI Use in
Teaching Veterinary Anatomy and the Correlation with Visual Spatial Ability: A Student Survey and Mental Rotations Test. Journal of veterinary medical education, 2017, pp. 1-10.

DOS REIS, Daniela de Alcântara Leite; GOUVEIA, Beatriz Laura Rojas; DE ALC NTARA, Brenda Mendonça; SARAGIOTTO, Brunna Pereira; BAUMEL, Érica Esther Domanski; FERREIRA, Janaina Santos; ROSA JÚNIOR, José Carlos; DE OLIVEIRA, Francileusa Delys; SANTOS, Paulo Ramos da Silva \& DE ASSIS NETO, Antônio Chaves. Biomodelos Ósseos Produzidos por Intermédio da Impressão 3D: Uma Alternativa Metodológica no Ensino da Anatomia Veterinária. Revista de Graduação USP, v. 2, n. 3, 2017, pp. 47-53.

DYCE, Keith M.; WENSING, Cornelius J. G.; SACK, Wolfganf $\mathrm{O}$. Tratado de anatomia veterinária. Elsevier Brasil, 2010.

FRANCESCHINI, Licurgo José; CARVALHO, Vilma Clóris de. Exposição ao formaldeído em anatomia: um risco de saúde ocupacional?. Rev. bras. ciênc. morfol, v. 10, n. 2, 1993, pp. 137-41.

FRUHSTORFER, Birgit H.; PALMER, J.; BRYDGES, S. \& ABRAHAMS, P. H. The use of plastinated prosections for teaching anatomy-the view of medical students on the value of this learning resource. Clinical Anatomy, v. 24, n. 2, 2011, pp. 246-252.

GARG, Amit X.; NORMAN, Geoff; SPEROTABLE, Lawrence. How medical students learn spatial anatomy. The Lancet, v. 357, n. 9253, 2001, pp. 363-364.

GARG, Amit X.; NORMAN, Geoffrey R.; EVA, Kevin W.; SPERO, Lawrence; SHARAN, Sumit. Is there any real virtue of virtual reality?: The minor role of multiple orientations in learning anatomy from computers. Academic Medicine, v. 77, n. 10, 2002, pp. S97-S99.

GROSS, M. Melissa; WRIGHT, Mary C.; ANDERSON, Olivia S. Effects of image-based and text-based active learning exercises on student examination performance in a musculoskeletal anatomy course. Anatomical sciences education, v. 10, n. 5, 2017, pp. 444-455.

GUMMERY, Erica; COBB, Kate A.; MOSSOP, Liz H. \& COBB, Malcolm A. Student Perceptions of Veterinary Anatomy Practical Classes: A Longitudinal Study. Journal of veterinary medical education, 2017, pp. 1-14.

HARRISON, Jon F.; NICHOLS, Jennifer S.; WHITMER, Allison G. Evaluating the impact of physical renovation, computerization, and use of an inquiry approach in an undergraduate, allied health human anatomy and physiology lab. Advances in physiology education, v. 25, n. 4, 2001, pp. 202-210.

HISLEY, Kenneth C.; ANDERSON, Larry D.; SMITH, Stacy E.; KAVIC, Stephen M. \& TRACY, J. Kathleen. Coupled physical and digital cadaver 
dissection followed by a visual test protocol provides insights into the nature of anatomical knowledge and its evaluation. Anatomical sciences education, v. 1, n. 1, 2008, pp. 27-40.

JAFFAR, Akram Abood. YouTube: An emerging tool in anatomy education. Anatomical sciences education, v. 5 , n. 3, 2012, pp. $158-164$.

JOHN, Nigel W. The impact of Web3D technologies on medical education and training. Computers of Education, v. 49, n. 1, 2007, pp. 19-31.

LATORRE, Rafael M.; GARCÍA-SANZ, Mary P.; MORENO, Matilde; HERNÁNDEZ, Fuensanta; GIL, Francisco; LÓPEZ, Octavio; AYALA, Maria D.; RAMÍREZ, Gregorio; VÁZQUEZ, Jose M.; ARENCIBIA, Alberto \& HENRY, Robert W. How useful is plastination in learning anatomy?. Journal of Veterinary Medical Education, v. 34, n. 2, 2007, pp. 172-176.

LIMBERGER, Daniela Cristina Haas. Processos de recuperação, reuso e destinação do formol em laboratório de anatomia. Dissertação (Mestrado em Engenharia) - Universidade Federal de Santa Maria, Santa Maria, 2011.

MAHMUD, Waqas; HYDER, Omar; BUTT, Jamaal \& AFTAB, Arsalan. Dissection videos do not improve anatomy examination scores. Anatomical sciences education, v. 4, n. 1, 2011, pp. 16-21.

MARTINSEN, Siri; JUKES, Nick. Towards a humane veterinary education. Journal of Veterinary Medical Education, v. 32, n. 4, 2005, pp. 454-460.

MATERA, Júlia Maria. Nível de aprendizado nos métodos substitutivos ao uso de animais no ensino. Revista CFMV, v. 15, n. 46, 2009, pp. 01-84.

MCCUSKEY, Robert S.; CARMICHAEL, Stephen W.; KIRCH, Darrell G. The importance of anatomy in health professions education and the shortage of qualified educators. Academic Medicine, v. 80, n. 4, 2005, pp. 349-351.

McMENAMIN, Paul G.; QUAYLE, Michelle R.; McHENRY, Colin R. \& ADAMS, Justin W. The production of anatomical teaching resources using three-dimensional (3D) printing technology. Anatomical sciences education, v. 7, n. 6, 2014, pp. 479-486.

NACIONAL OSSOS. Linha Veterinária. Disponível em: 〈http://ossos.com.br/loja/veterinaria.html〉. Acesso em 15 jan. 2018.

NEMANIC, Sarah; MILLS, Serena; VIEHDORFER, Matt; CLARK, Terri \& BAILEY, Mike. The Effectiveness of a 3D Computerized Tutorial to Enhance Learning of the Canine Larynx and Hyoid Apparatus. Journal of veterinary medical education, v. 43, n. 3, 2016, pp. 243-254.

NETO, Raul Antonio Fragoso; BIGONI, Paulo Sérgio. Substituição do formaldeído pela glicerina na conservação de preparações anatômicas. Revista Laborativa, v. 3, n. 1, 2014, pp. 75-87.
OLIVEIRA, Fabrício Singaretti. Assessing the effectiveness of 30\% sodium chloride aqueous solution for the preservation of fixed anatomical specimens: a 5-year follow-up study. Journal of anatomy, v. 225, n. 1, 2014, pp. 118-121.

OLIVEIRA, Fabrício Singaretti. Técnica alternativa de conservação de cadáveres animais. 2017. Disponível em: <http://unan.unesp.br/destaques/25768/tecnica-alternativa-de-conservacao-de-cadaveres-animais\&pagina $=1>$. Acesso em 17 fev. 2018.

PETTO, Andrew; FREDIN, Zachary; BURDO, Joseph. The use of modular, electronic neuron simulators for neural circuit construction produces learning gains in an undergraduate anatomy and physiology course. Journal of Undergraduate Neuroscience Education, v. 15, n. 2, 2017, pp. A151.

PREECE, Daniel; WILLIAMS, Sarah B.; LAM, Richard \& WELLER, Renate. "Let's get physical": advantages of a physical model over 3D computer models and textbooks in learning imaging anatomy. Anatomical sciences education, v. 6, n. 4, 2013, pp. 216-224.

RAFFAN, Hazel; GUEVAR, Julien; POYADE, Matthieu \& REA, Paul M. Canine neuroanatomy: Development of a 3D reconstruction and interactive application for undergraduate veterinary education. PloS one, v. 12, n. 2, 2017, pp. e0168911.

RINALDI, Vera D.; LORR, Nancy A.; WILLIAMS, Kimberly. Evaluating a technology supported interactive response system during the laboratory section of a histology course. Anatomical sciences education, v. 10, n. 4, 2017, pp. 328-338.

RODRIGUES, Hidegardo. Técnicas anatômicas. In: Técnicas anatômicas. 3. ed. Vitória, ES: [s.n.], 2005.

SABER, A. S.;SHOGHY, K. M. \&MOHAMMED, S. A. Plasticine Modeling as Alternative in Teaching Veterinary Anatomy. J. Vet. Anat. Vol, v. 9, n. 1, 2016, pp. 47-61.

SALTARELLI, Andrew J.; ROSETH, Cary J.; SALTARELLI, William A. Human cadavers vs. multimedia simulation: a study of student learning in anatomy. Anatomical sciences education, v. 7, n. 5, 2014, pp. 331-339.

SAXENA, Varun; NATARAJAN, Pradeep.; O'SULLIVAN, Patricia S. \& JAIN, Sharad. Effect of the use of instructional anatomy videos on student performance. Anatomical sciences education, v. 1, n. 4, 2008, pp. 159-165.

SCHOENAU, Luciana Silveira Flores; SCHOENAU, William. The production of videoclasses as a learning tool to the animal anatomy discipline. Electronic Journal of Management, Education and Environmental Technology (REGET), v. 12, n. 12, 2013, pp. 2560-2567.

SCHOENFELD-TACHER, Regina M.; HORN, 
Timothy J.; SCHEVIAK, Tyler A.; ROYAL, Kenneth D. \& HUDSON, Lola C. Evaluation of 3D Additively Manufactured Canine Brain Models for Teaching Veterinary Neuroanatomy. Journal of veterinary medical education, v. 44, n. 4, 2017, pp. 612-619.

SEIXAS-MIKELUS, Stéfanie A.; ADAL, Adaffaras; KESAVADAS, Thenkurussi; BAHETI, Ankur; SRIMATHVEERAVALLI, Govindarajan; HUSSAIN, Abid; CHANDRASEKHAR, Rameela; WILDING, Gregory E. \& GURU, Khurshid A. Can image-based virtual reality help teach anatomy?. Journal of endourology, v. 24, n. 4, 2010, pp. 629-634.

SIMÃO, Vitor Tadeu Cunha; DE MELO, Maria Isabel Vaz. Inovação Tecnológica no ensino da anatomia veterinária. Sinapse Múltipla, v. 4, n. 1, 2015, pp. 63.

SIVIERO, Fábio; DE OLIVEIRA, Sérgio Ferreira. Modernização do Ensino de Biologia Tecidual. Revista de Graduação USP, v. 1, n. 1, 2016, pp. 35-40.

SMITH, Claire F.; TOLLEMACHE, Nicholas; COVILL, Derek \& JOHNSTON, Malcolm. Take away body parts! an investigation into the use of 3D-printed anatomical models in undergraduate anatomy education. Anatomical sciences education, 2017.

TEIXEIRA FILHO, Althen; GUARENTI, V. D. P. J.; TEIXEIRA, A.; CARAMBULA, S. F.; CRUZATI, A. \& BRUCKER, P. F. The cryodehydration technique applied to cavitary and parenchymatous viscera. Braz.j. morphol. sci, v. 13, n. 2, 1996, pp. 177-80.
TEIXEIRA, Althen; TEIXEIRA FILHO, Althen; GUARENTI, Vicente De Paula J. Desidrataçäo de músculos no preparo de peças anatômicas. Rev. bras. ciênc. morfol, v. 7, n. 2/1, 1991, pp. 45-7.

THEORET, Christine Laura; CARMEL, ÉricNorman; BERNIER, Sonia. Why dissection videos should not replace cadaver prosections in the gross veterinary anatomy curriculum: Results from a comparative study. Journal of veterinary medical education, v. 34, n. 2, 2007, pp. 151-156.

TOPPING, Daniel B. Gross anatomy videos: Student satisfaction, usage, and effect on student performance in a condensed curriculum. Anatomical sciences education, v. 7, n. 4, 2014, pp. 273-279.

VIEHDORFER, Matt; NEMANIC, Sarah; MILLS, Serena \& BAILEY, Mike. Virtual dog head: using 3D models to teach complex veterinary anatomy. In: ACM SIGGRAPH 2014 Studio. ACM, 2014. pp. 17.

WILSON, Adam B.; MILLER, Corinne H.; KLEIN, Barbie A.; TAYLOR, Melissa A.; GOODWIN, Michael; BOYLE, Eve K.; BROWN, Kirsten; HOPPE, Chantal \& LAZARUS, Michelle. A Meta-analysis of Anatomy Laboratory Pedagogies. Clinical Anatomy, 2017.

YAMMINE, Kaissar; VIOLATO, Claudio. A meta-analysis of the educational effectiveness of three-dimensional visualization technologies in teaching anatomy. Anatomical sciences education, v. 8, n. 6, 2015, pp. 525-538. 